\title{
A NEW PARALLEL EM ALGORITHM WITH THE OPTIMAL DATA REPLICATION ON A HYPERCUBE MULTIPROCESSOR FOR 3D PET IMAGE RECONSTRUCTION
}

\author{
Chung-Ming Chen* and Soo-Young Lee ${ }^{\dagger}$ \\ * Center for Biomedical Engineering, National Taiwan University, Taiwan \\ † School of Electrical Engineering, Cornell University, Ithaca, NY 14853
}

\begin{abstract}
In this paper, we present a new parallel EM algorithm with the optimal data replication on a hypercube multiprocessor. Although data replication has been frequently used to reduce data sharing overhead, none of the parallel EM algorithms have attempted to optimize the data replication. To maximize efficiency of the proposed parallel EM algorithm, data replication have been optimized through strict mathematical formulation and analysis in this study. To verify the theoretical analyses, we have implemented the proposed parallel EM algorithm on an Intel iPSC/860 and the experimental results are shown to be consistent with the theoretical results.
\end{abstract}

Keywords: Parallel EM Algorithms, Optimal Data Replications, Hypercube

\section{Introduction}

Positron Emission Tomography (PET) is an imaging modality to visualize the spatial distribution of positronemitting radioisotopes. By labeling active body constituents with positron emitters, PET has the unique capability to study in vivo physiological and metabolic functions in the human body. However, due to several practical considerations, PET has not been widely used in the medical environment. One of the major problems is the long image reconstruction time.

While image reconstruction may be sped up by varieties of approaches, it is generally believed that the most costeffective approach would be parallel processing. In the attempt to make 3D PET feasible for practical use, in this paper we present a new parallel EM algorithm on a hypercube message-passing multiprocessor. An EM algorithm is an iterative reconstruction algorithm first proposed by Shepp and Vardi [1]. The EM algorithms have received a great interest in the past decade since they promise to converge to an estimate with the maximum likelihood and tend to reduce the statistical noise artifact.

There have been several parallel EM algorithms proposed for various types of parallel systems $[2,3,4,5]$. However, none of them have achieved the optimal parallelization efficiency on a message-passing system. The common problem involved in these parallel EM algorithms is that the data sharing overhead is not minimized. While the most widely used approach to reducing the data sharing overhead on a message-passing system is to replicate the shared data, none of these parallel EM algorithms have attempted to employ the optimal data replication.

Our approach to minimizing data sharing overhead is based on the optimal data replication. Through strict mathematical formulation and analysis, we have derived the optimal group sizes and shown that the integration and broadcasting algorithms as well as the scheduling of shared data accesses, which support the optimal data replication, are optimal. To verify our theoretical analyses, we have implemented the proposed parallel EM algorithm on an Intel iPSC/860 with 16 PEs and experimental results consistent with the theoretical results have been obtained.

\section{Parallel EM Algorithm}

The EM algorithm employed in this study is the one proposed by Shepp and Vardi $[1]$ since it has the similar algorithmic structure as most of the other EM algorithms. From the viewpoint of parallelization, the Shepp and Vardi's EM algorithm is an ideal representative for these EM algorithms. An iteration of the EM algorithm proposed by Shepp and Vardi may be decomposed into four steps as follows:
(1) $\tilde{n}=\lambda^{\text {old }} P$
(2) $\varepsilon(t)=n(t) / \tilde{n}(t)$, for all $t$,
(3) $\delta=P \varepsilon^{T}$,
(4) $\lambda^{\text {new }}(b)=\delta(b) \lambda^{\text {old }}(b)$, for all $b$.

where $n$ is the projection data, $\lambda$ the image to be reconstructed, and $P$ the probability matrix. Also, $b$ denotes the $b$ th box (voxel) and $t$ denotes the $t$ th tube.

Two issues should be considered in parallelization of the EM algorithm. One is the load balancing and the other is the data sharing overhead. While a well balanced load distribution is not difficult to achieve [3], data sharing is inevitable in parallelizing the EM algorithm. To reduce the need for data sharing, in the proposed parallel EM algorithm, the tasks are partitioned as in the partition-by-tube scheme proposed in [3], which is one of our early works. This partitioning scheme not only can achieve a balanced computational load distribution but also can confine data sharing in steps 3 and 4.

The data sharing overhead at steps 3 and 4 is minimized by optimizing data replication. First, all PEs are divided into groups and all groups have the same number of PEs. 
The PEs in a group form a subcube. Then, the shared data, $\delta$, are replicated as follows. Each group of PEs share a copy of shared data. The shared data are divided in such a way that all PEs have the same amount of shared data in their own local memory. Moreover, each copy of shared data is altocated such that each PE needs to access the local memories of all PEs in the same group the same number of times. It is assumed that the communication link setup time is negligible due to either a large enough block size or a very fast link setup.

During step 3, all PEs store the computed partial results, $P(b, t) \varepsilon(t)$, in their own local memories. At the end of step 3, each PE sends the partial results to the associated PEs and updates the shared data. If there are data replicated, the replicated shared data will be integrated and broadcast to ensure data coherence by using the $R e$ verse Exchange and Exchange algorithms [4] which are optimal on a hypercube.

For each PE, the overall data sharing time is composed of three parts, namely, the times for sending the partial results to all other PEs, updating the shared data using the partial results and integrating and broadcasting the replicated data. Given a group size, since the second part is a constant and the third part is optimal, the overall data sharing time is minimized if the first part, i.e., the time for sending partial results, is minimized. To minimize the first part, we have developed a scheduling algorithm, which has been mathematically shown to be optimal, for sending the partial results from all PEs to all other PEs. For ease of presentation, denote the minimized data sharing overhead for a give group size, $2^{x}$, as $T(x)$.

To derive the optimal group size which minimizes the overall data sharing time, $\partial T(x) / \partial x=0$ is solved for the optimal $x$. Limited by the space, the derivation of the optimal group size and the scheduling algorithm for sending partial results are not provided in this paper which, however, may be found in [6]. Also, since the formulas of the optimal group sizes vary with the communication capability of the parallel systems employed, we will provide the optimal group size only for the class of hypercube multiprocessors which has the same communication capability as the one available to us. The feature of this class is that only one link of each PE can carry out communication at a time, which is defined as single-channel link capabil$i t y$. For the optimal group sizes of more general types of hypercube multiprocessors, please refer to [6].

Proposition 1 The optimal group size on a binary $n$ cube with single-channel link capability is

$$
\log _{2} \frac{\left(2+T_{m}\right) \ln 2}{N_{a} T_{x}}
$$

where $\ln$ is the natural logarithm.

In Proposition $1, T_{m}$ is the time for updating a shared datum with one partial result, $N_{a}$ the average number of shared data access associated with each shared datum, and $T_{x}$ the time for transferring one datum between two adjacent PEs.

\section{Results and Discussions}

To verify the theoretical analysis results, we have implemented the proposed parallel EM algorithm on an iPSC/860 with 16 PEs. Although iPSC/860 virtually allows all links to perform communication simultaneously, in practice, each PE may transmit data only through one link at a time due to the limited capability of the DirectConnect Module on the iPSC/860. Different sizes of PET systems and images as well as various CPU capability have been simulated to see the effect of $T_{m}$ and $N_{a}$. As an example, the performances attained by all possible group sizes for various $T_{m}$ and $N_{a}$ are shown in Fig. 1 .

Although the theoretical results are not shown in this paper due to the limited space, they are quite consistent with the experimental results [6]. From these results, we can see that using the optimal group size may reduce the data sharing overhead substantially than using an improper group size, e.g., compared to the frequentlyused approach, i.e., replicating the entire shared data in each PE. Moreover, it should be emphasized that the proposed parallel EM algorithm always provides the best performance among those parallel EM algorithms using balanced computational load distributions and data replication on a hypercube with negligible link setup time.

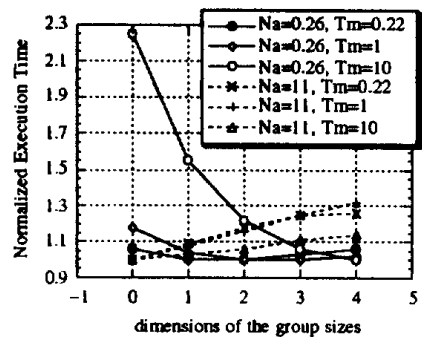

Figure 1: Normalized execution time on a binary 4-cube

\section{References}

[1] L. A. Shepp and Y. Vardi, "Maximum likelihood reconstruction for emission tomography," IEEE Thans. Med. Imaging, vol. MI-1, pp. 113-122, oct. 1982.

[2] F. U. Rosenberger, D. G. Politte, G. C. Johns, and C. E. Molnar, "An efficient parallel implementation of the EM algorithm for PET image reconstruction utilizing transputers," in 1990 Nuclear Science Symposium Conference Record, Oct. 1990.

[3] C. M. Chen, S.-Y. Lee, and Z. H. Cho, "Parallelization of the EM algorithm for 3D PET image reconstruction," IEEE Trans. Med. Imaging, vol. 10, pp. 513-522, Dec. 1991.

[4] C. M. Chen and S.-Y. Lee, "On parallelizing the EM algorithm for PET image reconstruction." to appear in IEEE Trans. Parallel and Distributed Systems.

[5] G. T. Herman, D. Odhner, K. D. Toennies, and S. A. Zenios, "A parallelized algorithm for image reconstruction from noisy projections," in Large-Scale Numerical Optimization, Oct. 1989.

[6] C. M. Chen, On Miniminzing Data Sharing Overhead for Large-Scale Data-Parallel Algorithms: Replication and Allocation of Shared Data. PhD thesis, Cornell University, Ithaca, New York, 1993. 